\title{
Microstrip Patch Antenna Bandwidth Enhancement Using AMC/EBG Structures
}

\author{
R. C. Hadarig, M. E. de Cos, and F. Las-Heras \\ Area de Teoría de la Señal y Comunicaciones, Departamento de Ingeniería Eléctrica, Universidad de Oviedo, Edificio Polivalente, \\ Modulo 8, Campus Universitario de Gijón, Asturias, 33203 Gijón, Spain
}

Correspondence should be addressed to M. E. de Cos, medecos@tsc.uniovi.es

Received 11 July 2011; Revised 19 September 2011; Accepted 22 September 2011

Academic Editor: Zhongxiang Q. Shen

Copyright ( 92012 R. C. Hadarig et al. This is an open access article distributed under the Creative Commons Attribution License, which permits unrestricted use, distribution, and reproduction in any medium, provided the original work is properly cited.

A microstrip patch antenna with bandwidth enhancement by means of artificial magnetic conductor (AMC)/electromagnetic band-gap structure (EGB) is presented. The electrical characteristics of the embedded structure are evaluated using MoM simulations. The manufactured prototypes are characterized in terms of return loss, gain, and radiation pattern measurements in an anechoic chamber.

\section{Introduction}

Microstrip patch antennas offer an attractive solution to compact and ease-low-cost design of modern wireless communication systems due to their many advantages as light weight and low volume, low profile, planar configuration which can be easily made conformal to host surface, low fabrication cost, and the capability of obtaining dual and triple frequency operations. When mounted on rigid surfaces microstrip patch antennas are mechanically robust and can be easily integrated with microwave integrated circuits (MICs).

However, microstrip patch antennas suffer from a number of disadvantages as compared to conventional nonprinted antennas. Some of their major drawbacks are the narrow bandwidth, low gain, and surface wave excitation that reduce radiation efficiency. To overcome one of their more critical restrictions, narrow bandwidth, several techniques can be used [1]. First of all, a thicker substrate with a low dielectric constant or a ferrite composition provides a wider bandwidth but the first approach leads to no low-profile designs and increased in size, whereas the second solution is expensive. Secondly, noncontacting feeding methods such as proximity/aperture coupled can be used to improve the impedance bandwidth, but this is difficult to fabricate. Another possibility is multiresonator stack configuration with the inconvenient of resulting large thickness prototype $[2,3]$.
The surface waves can be minimized using electromagnetic band-gap structures whereas for obtaining a high gain antenna an array configuration for the patch elements is needed.

The research in the field of electromagnetic band-gap structures has become attractive in the antenna community and is considered to be a key technology for improving microstrip patch antenna performances [4-6]. The use of metamaterials, such as the frequency selective surfaces (FSS) [79] is an alternative to face antennas and microwave circuit problems and can provide either EBG or AMC behavior. In previous works, several narrow band antennas have been mounted on EBG/AMC structures [10-18].

Depending on the intended application, the $2.4 \mathrm{GHz}$ frequency band can be used, for example, for wireless communications at $2.45 \mathrm{GHz}$ or for RFID systems at $2.48 \mathrm{GHz}$. In this paper, the main goal is to improve the bandwidth and the radiation properties of a microstrip patch antenna in the $2.48 \mathrm{GHz}$ band using two different approaches: combination of the patch antenna with an EBG structure in the same layer and combination of the patch antenna with an AMC structure in two different layers. The aim of this work is challenging because two resonant structures are involved and when integrated together their resonant behavior is mutually influenced. Firstly, the design of a microstrip patch antenna, henceforth referred as patch antenna, is shown followed by an adaptation of an AMC design recently presented by 


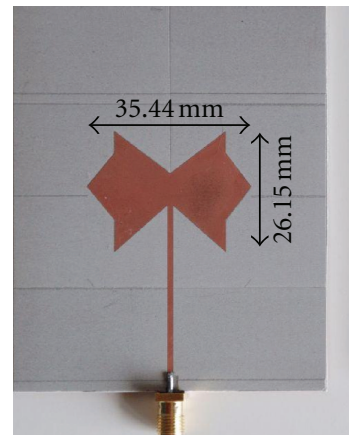

(a)

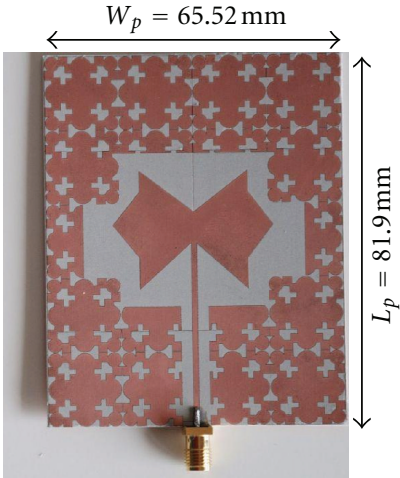

(b)

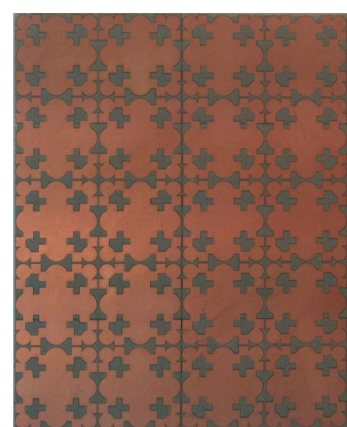

(c)

Figure 1: Manufactured prototypes: (a) Patch antenna, (b) Patch antenna-EBG, (c) Planar AMC.

the authors to operate at $2.48 \mathrm{GHz}$. Then, the patch antenna is placed above the AMC. This combination will be henceforth referred as Patch antenna-AMC. Secondly, the AMC structure is modified to act as an EBG at a frequency close to the patch antenna resonance frequency. Finally the EBG is combined with the patch antenna on the same layer, resulting in a design with a uniplanar feature and reduced cost. This combination will be henceforth referred as patch antennaEBG. Return loss, gain, and radiation patterns of the three prototypes (all having the same dimensions) are analyzed based on measurements in an anechoic chamber.

\section{Microstrip Antenna Design}

The microstrip patch antenna is a narrow band design. In this work, the patch antenna suitable for RFID applications at $2.48 \mathrm{GHz}$ is designed using ROGER3010 substrate with a thickness of $1.27 \mathrm{~mm}$, relative dielectric permittivity $\varepsilon_{r}=$ 10.2 , and loss tangent of 0.0023 . The geometry of the patch antenna with its dimensions is shown in Figure 1(a). The characteristic impedance of the transmission line is $50 \Omega$. The antenna design has been carried out by a set of methodof-moments (MoM) simulations with commercial software [19]. From Figure 4, it can be extracted that the simulated operating bandwidth of the patch antenna is $20 \mathrm{MHz}$.

2.1. AMC Characterization. An adaptation of the AMC previously designed by the authors [20] is carried out shifting the resonant frequency to $2.48 \mathrm{GHz}$. Based on the BlochFloquet theory and on the finite element method (FEM), a single cell of the lattice with periodic boundary conditions (PBCs) on its four sides is simulated in order to obtain the frequency band where the periodic structure acts as an AMC. The phase of the reflection coefficient on the AMC surface is computed using a uniform incident plane wave (see Figure 2(a)). Depending on the unit cell geometry together with the substrate's thickness and relative dielectric permittivity, the resonant frequency and the bandwidth of the structure can be tuned. The unit cell dimensions are $W \times W=$ $16.93 \times 16.93 \mathrm{~mm}^{2}$ and its geometry exhibits four symmetry planes. The simulated reflection phase of normally incident plane wave on the AMC surface versus frequency is represented in Figure 2. The AMC resonant frequency is $2.48 \mathrm{GHz}$, and the AMC operation bandwidth is approximately $130 \mathrm{MHz}$ (5.24\%) (see Figure 2(b)). The structure exhibits several advantages such as uniplanar feature since neither multilayer substrate no via holes are required, simplifying the implementation and reducing its costs.

2.2. EBG Characterization. The periodic structure can be characterized as EBG using the suspended strip method [21, 22] (See Figure 3). A suspended strip line over the $4 \times 4$ cell arrangement is used to test the transmission response of the electromagnetic waves. The strip height is $0.02 \lambda$. The structure will block the transmission of power along the strip line for frequencies within the band gap region and a noticeable reduction in $S_{21}$ can be observed at a certain frequency band. The band-gap of the EBG lattice is designed to be adjacent to the frequency band of the patch antenna, so that when integrating the two structures on the same layer, their resonances couple each other, and, as a result, a wider bandwidth will be generated without disturbing other characteristics of the patch antenna such as the radiation pattern. The dimension of the unit cell in the case of EBG characterization is $W_{2}=16.38 \mathrm{~mm}$. The simulated band gap of the EBG structure closer to the patch antenna bandwidth is $45 \mathrm{MHz}$ around $2.5 \mathrm{GHz}$ (see Figure 4).

\section{Microstrip Patch Antenna Combined with AMC/EBG}

3.1. Patch Antenna Placed above the AMC Structure. A $4 \times$ 5 cells planar AMC structure is placed as patch antenna ground plane [23] (see Figure 5) in order to analyze if the antenna's bandwidth and the radiation properties can be improved. The antenna is fixed to the AMC structure (see Figure $1(\mathrm{c})$ ) by a $0.1 \mathrm{~mm}$ double-sided nonconducting adhesive tape. The microstrip patch antenna bandwidth is $20 \mathrm{MHz}$ whereas the AMC operation bandwidth is $130 \mathrm{MHz}$, having each one the same resonance frequency, $2.48 \mathrm{GHz}$, and the same dimensions. However, for combining the two structures, the antenna's ground plane has been removed and is placed above the AMC. As a consequence the antenna's 


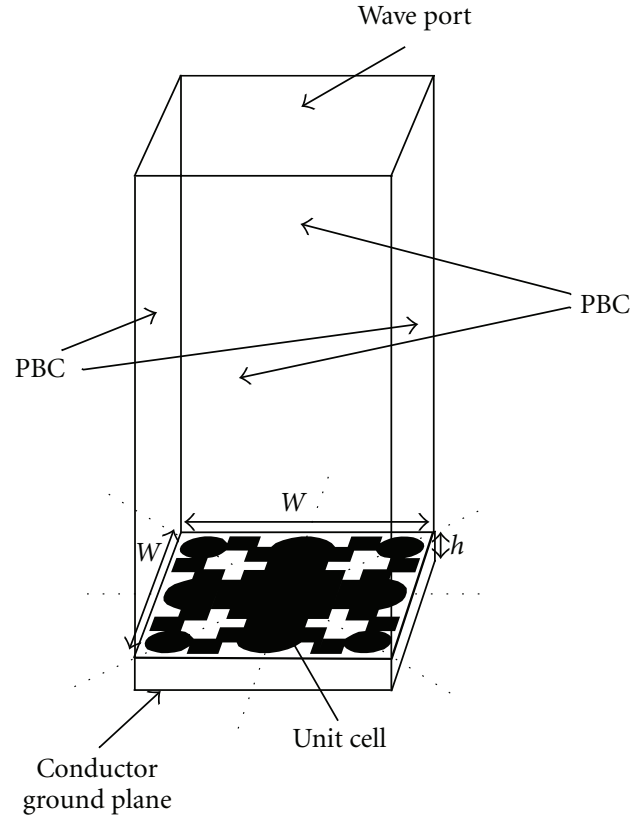

(a)

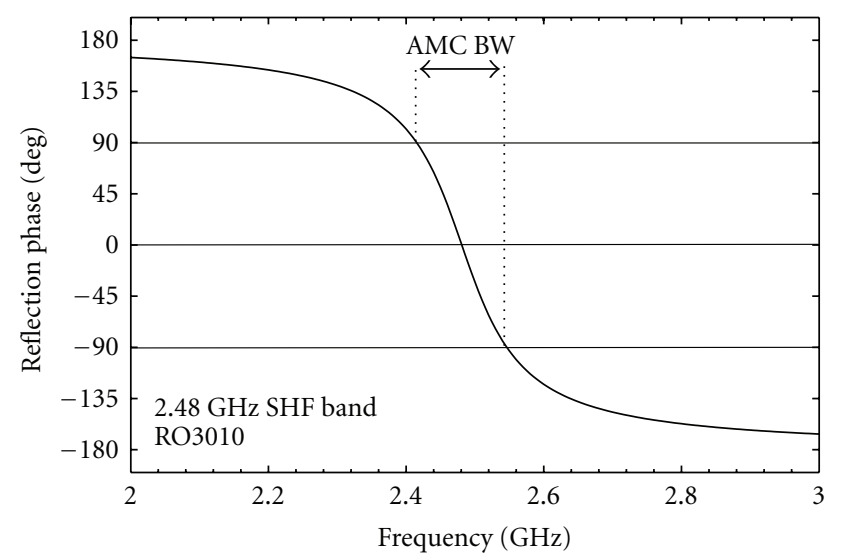

(b)

FIGURE 2: AMC unit cell: (a) reflection phase simulation setup, (b) phase of the reflection coefficient on the AMC surface.

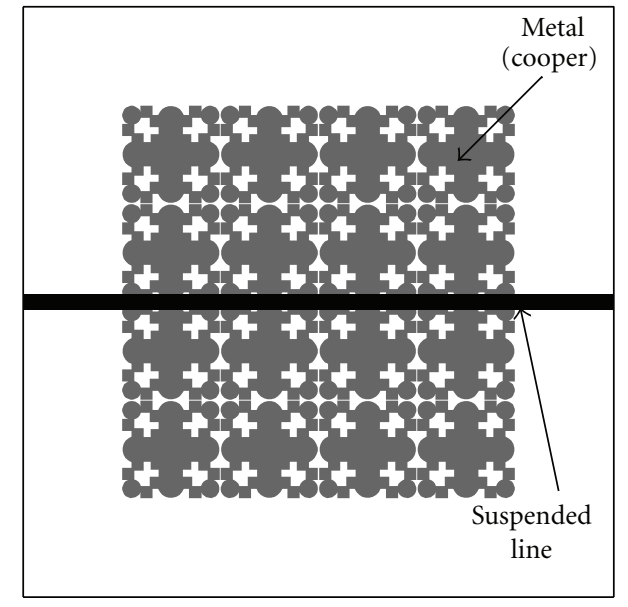

Figure 3: Schematic of suspended line above EBG surface (top view).

resonance frequency decreases due to capacitive effects for those frequencies within the AMC bandwidth. A resonance is obtained in the AMC bandwidth and outside this band the antenna behaves as if its substrate thickness had doubled. Merging both effects, the combined structure resonates in a bandwidth wider than the microstrip patch antenna alone, but narrower than the AMC bandwidth. As disadvantage, the thickness of the combined structure is increased. If the dielectric substrate thickness of the patch antenna doubles, the resulting bandwidth $(30 \mathrm{MHz})$ is narrower compared to the bandwidth of patch antenna-EBG and patch antennaAMC prototypes.

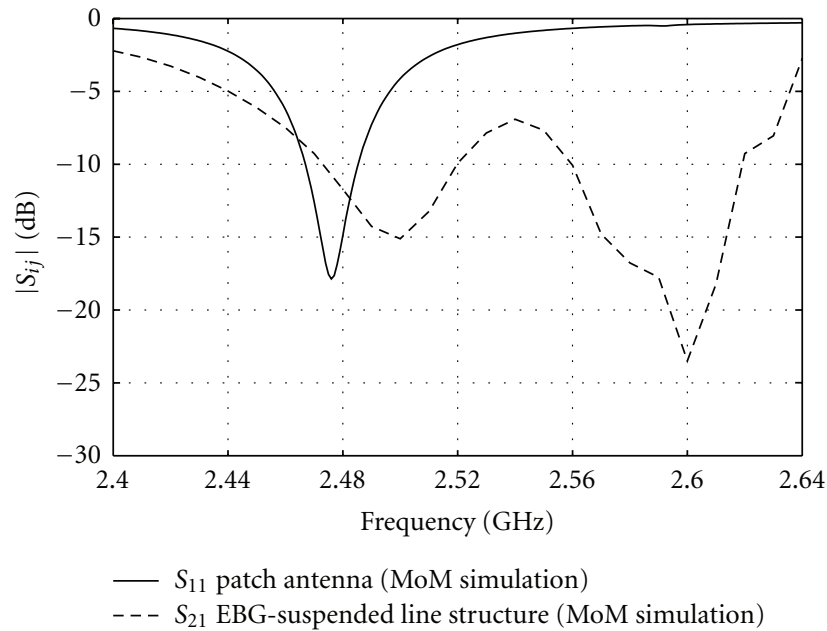

FIGURE 4: Resonances to be coupled in order to achieve bandwidth enhancement.

3.2. Patch Antenna Surrounded by the EBG Structure. In order to suppress the surface waves and to increase the bandwidth by means of coupled resonators effect, the EBG lattice is arranged around the patch, forming a uniplanar design [24]. As it has been already mentioned in Section 2.2, the resonance frequency of both structures (patch antenna and EBG structure) is mutually influenced, and depending on the frequency difference between them and the unit-cell arrangement around the patch antenna, the resulting resonance frequency changes. The frequencies included on the patch antenna's bandwidth are adjacent to the ones included on the lower band gap. The selected EBG arrangement with 


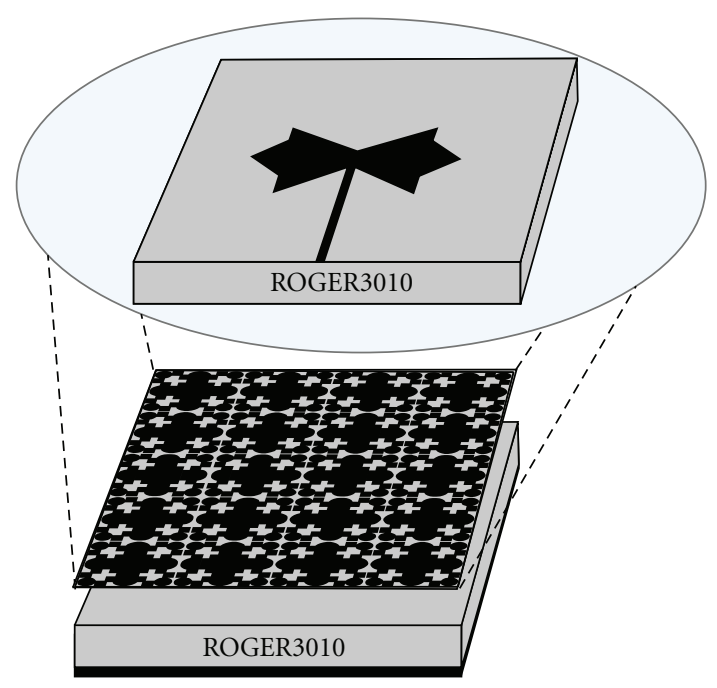

FIgURE 5: Patch antenna-AMC prototype layout.

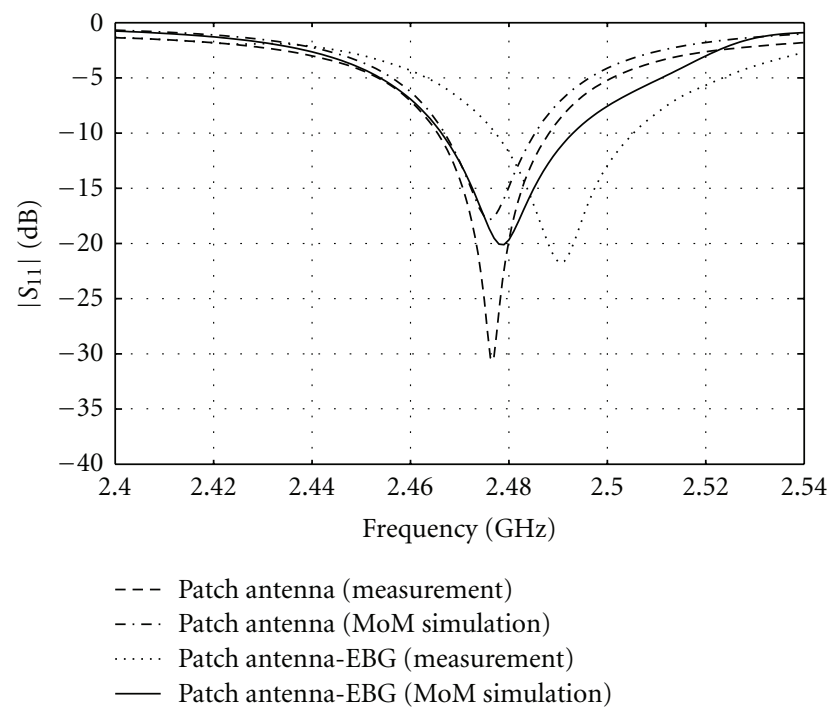

FIGURE 6: Simulation and measurement comparison between the prototypes: patch antenna and patch antenna-EBG.

respect to the antenna is a tradeoff between performance and size. The dimensions of the final structure (Figure 1(b)) are $W p=65.52 \mathrm{~mm}$ and $L_{p}=81.90 \mathrm{~mm}$.

\section{Results}

Prototypes of the patch antenna, patch antenna placed above the AMC surface, and patch antenna surrounded by the EBG cells have been manufactured using laser micromachining. The return losses of each manufactured prototype have been measured. As it can be observed in Figure 6 the measured operating bandwidth of the patch antenna is $23 \mathrm{MHz}$. The difference in bandwidth between simulations $(20 \mathrm{MHz})$ and measurement $(23 \mathrm{MHz})$ results could be due to the fact that the commercial MoM software considers infinite extension

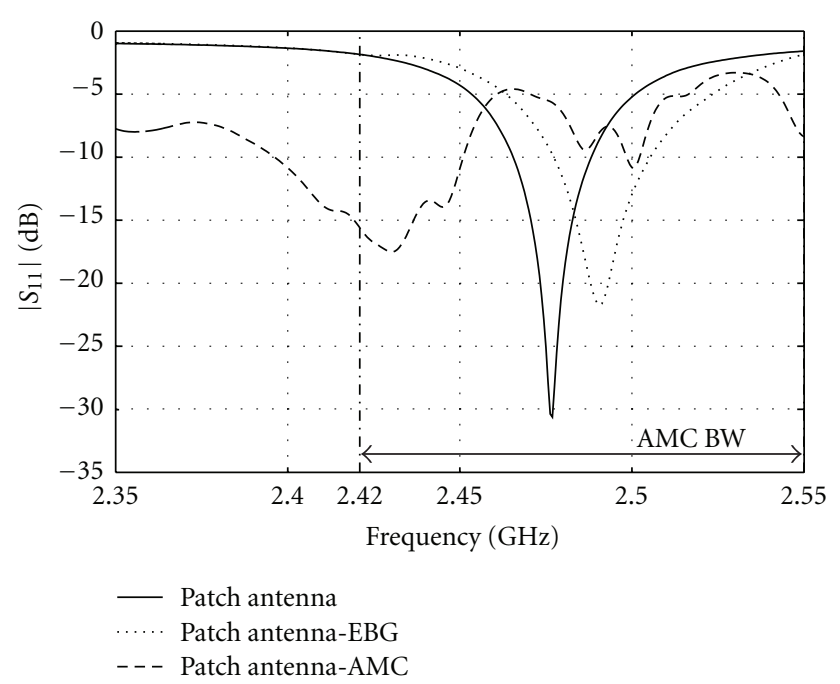

FIGURE 7: Measurement comparison between the prototypes: patch antenna, patch antenna-EBG, and patch antenna-AMC.

for the dielectric substrate, or even more likely due to manufacturing tolerances.

In the case of placing the antenna above the AMC surface the antenna resonance frequency is shifted downwards to $2.43 \mathrm{GHz}$ (see Figure 7) due to the capacitive effects that are generated between the two combined structures. Also, as the AMC structure has wider bandwidth than the patch antenna, the resulting prototype bandwidth increases to $46 \mathrm{MHz}$, meaning a $100 \%$ broader bandwidth (see Figure 7 ).

When the patch antenna is surrounded by one row of EBG cells the bandwidth increases 50\% (see Figure 7) due to the property of coupling the frequency bands of the two structures composing the prototype. It is remarkable that this $50 \%$ bandwidth improvement is achieved increasing neither the prototype size nor the thickness. The percentage bandwidth comparison of the three prototypes is presented in Table 1.

Measured radiation pattern cuts in the $\mathrm{E}$ and $\mathrm{H}$ planes of each manufactured prototype are presented in Figure 8. The patch antenna prototype exhibits copolarization-crosspolarization (CP-XP) ratio better than $25 \mathrm{~dB}$ (see Table 2), whereas for the patch antenna-EBG prototype the (CP-XP) ratio and the directivity are even increased. In measurements the gain of the patch antenna $(4.59 \mathrm{~dB})$ is preserved when the antenna is surrounded by one row of EBG cells (Table 1). From the simulation results, using EGB structures around the patch antenna its radiation efficiency increases, due to surface wave suppression property. However from measurement results it can be concluded that for this specific arrangement, the radiation efficiency is preserved (while improving bandwidth). The difference between simulations and measurements relies on the fact that the simulation method implemented by Momentum considers infinite dielectric under the finite EBG metallization but also the difference could be attributable to misalignments in the anechoic chamber. Radiation pattern properties of the patch antennaAMC prototype show a $(\mathrm{CP}-\mathrm{XP})$ ratio inferior to the other 


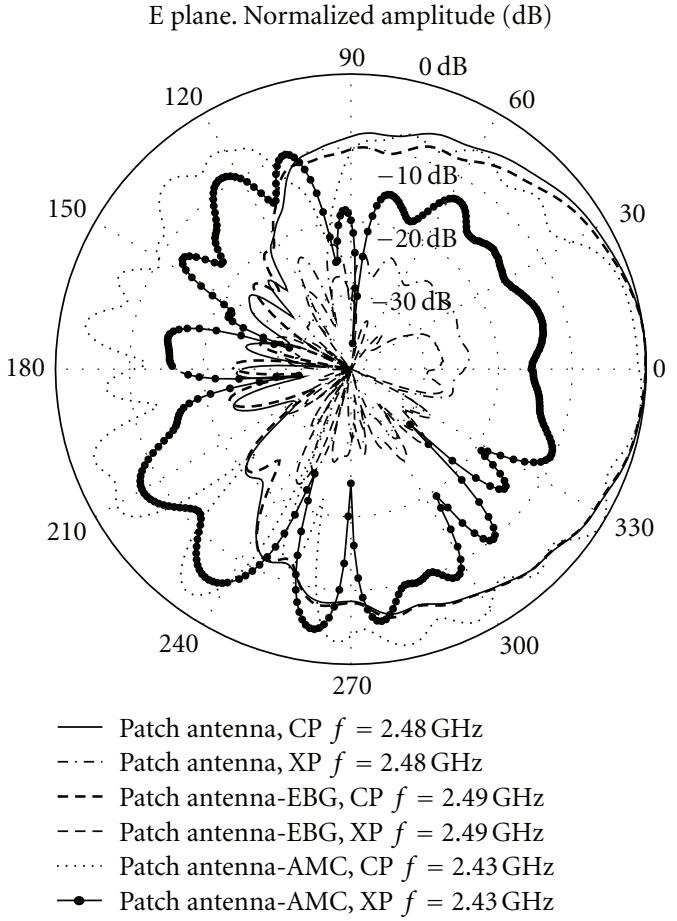

(a)

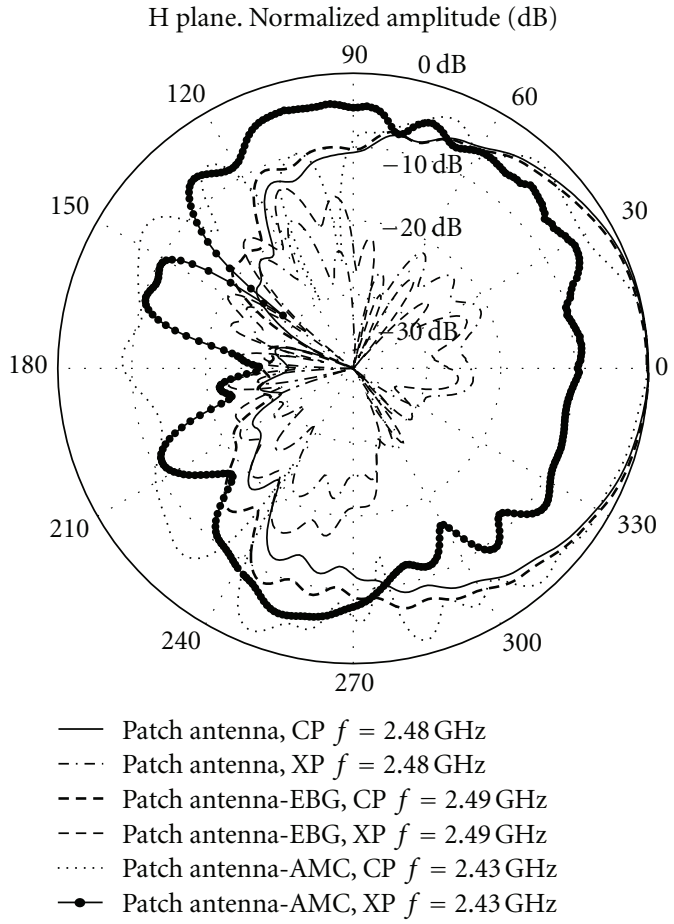

(b)

FIGURE 8: Measured radiation patterns of the prototypes: Patch antenna, patch antenna-EBG, and patch antenna-AMC.

TABLE 1: Comparison between the three designs.

\begin{tabular}{|c|c|c|c|c|c|c|c|}
\hline \multirow[t]{2}{*}{ Prototype } & \multirow{2}{*}{$\begin{array}{c}\text { Bandwidth }(\mathrm{MHz}) \\
\text { Meas. }\end{array}$} & \multicolumn{2}{|c|}{ Directivity (dB) } & \multicolumn{2}{|c|}{ Gain $(\mathrm{dB})$} & \multicolumn{2}{|c|}{ Radiation efficiency (\%) } \\
\hline & & Meas. & Sim. & Meas. & Sim. & Meas. & Sim. \\
\hline Patch antenna & $23(0.93 \%)$ & 7.33 & 5.95 & 4.59 & 4.29 & 53.21 & 68.23 \\
\hline Patch antenna-EBG & $34(1.37 \%)$ & 7.50 & 6.84 & 4.61 & 5.56 & 51.40 & 74.47 \\
\hline Patch antenna-AMC & $46(1.90 \%)$ & 6.72 & 8.52 & 0 & 0.79 & 21.28 & 16.86 \\
\hline
\end{tabular}

two prototypes and a gain close to $0 \mathrm{~dB}$. As the AMC does not have the ability to suppress the surface waves and the fact that the thicker the substrate, the stronger are the surface waves, the gain of the patch antenna-AMC prototype does not improve. Also as the CP-XP ratio is worst for patch antennaAMC than for the other prototypes, part of the energy could be radiated in other polarizations and backwards (as it is shown in Figure 8). In order to improve the gain, a gap between the antenna and the AMC surface could be used but this is technologically less advantageous. In addition, the microstrip patch antenna's gain and directivity can be increased when more rows or/and columns surround the prototype, so a trade-off between performance and size must be taken (the higher the number of unit cells in a periodic arrangement, the closer its behavior to an infinite EBG structure).

\section{Conclusions}

Bandwidth enhancement of microstrip patch antenna in the RFID SHF $2.48 \mathrm{GHz}$ band has been presented. Two different structures (AMC/EBG) have been combined with the same
TABLE 2: CP-XP ratio comparison.

\begin{tabular}{lcc}
\hline Prototype & $\begin{array}{c}\text { CP-XP ratio } \\
\text { (E plane, dB) }\end{array}$ & $\begin{array}{c}\text { CP-XP ratio } \\
(\text { H plane, dB) }\end{array}$ \\
\hline Patch antenna & 25.81 & 25.04 \\
Patch antenna-EBG & 30.43 & 28.79 \\
Patch antenna-AMC & 13.85 & 9.43 \\
\hline
\end{tabular}

microstrip patch antenna in order to characterize their joint performance. The prototypes have been manufactured and characterized based on measurements in an anechoic chamber. From the measurements of the two resulting prototypes, patch antenna-AMC and patch antenna-EBG, it can be concluded that both prototypes improve the bandwidth of the patch antenna. Due to the surface wave effect of the EBG structure, the patch antenna-EBG prototype shows better radiation properties increasing neither the prototype size nor the thickness. All the prototypes presented are robust, compact and do not require via holes, being compatible with planar fabrication technology. 


\section{Acknowledgments}

This work has been supported by the Ministerio de Ciencia e Innovación of Spain/FEDER under Projects TEC200801638/TEC (INVEMTA), and CONSOLIDER-INGENIO CSD2008-00068 (TERASENSE), by the Gobierno del Principado de Asturias (PCTI)/FEDER-FSE under Projects EQUIP08-06, FC09-COF09-12, EQUIP10-31, and PC10-06 (FLEXANT), by Grant BP10-039, and by Cátedra Telefónica, Universidad de Oviedo.

\section{References}

[1] R. Garg, I. Bhartia, I. Bahl, and A. Ittipiboon, Microstrip Antenna Design Handbook, Artech House, Boston, Mass, USA, 2001.

[2] G. Kumar and K. C. Gupta, "Directly coupled multiple resonator wide-band microstrip antenna," IEEE Transactions on Antennas and Propagation, vol. 33, no. 6, pp. 588-593, 1985.

[3] F. Yang, X. X. Zhang, X. Ye, and Y. Rahmat-Samii, "Wide-band E-shaped patch antennas for wireless communications," IEEE Transactions on Antennas and Propagation, vol. 49, no. 7, pp. 1094-1100, 2001.

[4] A. Pirhadi, F. Keshmiri, M. Hakkak, and M. Tayarani, "Analysis and design of dual band high directivity EBG resonator antenna using square loop FSS AS superstrate layer," Progress in Electromagnetics Research, vol. 70, pp. 1-20, 2007.

[5] E. Rajo-Iglesias, L. Inclán-Sánchez, and O. Quevedo-Teruel, "Back radiation reduction in patch antennas using planar soft surfaces," Progress In Electromagnetics Research Letters, vol. 6, pp. 123-130, 2009.

[6] Z. Duan, S. Qu, and Y. Hou, "Electrically small antenna inspired by spired split ring resonator," Progress In Electromagnetics Research Letters, vol. 7, pp. 47-57, 2009.

[7] F. Yang and Y. Rahmat-Samii, Electromagnetic Band-Gap Structures in Antenna Engineering, The Cambridge RF and Microwave Engineering Series, Cambridge University Press, Cambridge, Mass, USA, 2008.

[8] M. E. De Cos, F. L. Heras, and M. Franco, "Design of planar artificial magnetic conductor ground plane using frequencyselective surfaces for frequencies below $1 \mathrm{GHz}$," IEEE Antennas and Wireless Propagation Letters, vol. 8, pp. 951-954, 2009.

[9] O. Luukkonen, C. R. Simovski, and S. A. Tretyakov, "Grounded uniaxial material slabs as magnetic conductors," Progress in Electromagnetics Research B, no. 15, pp. 267-283, 2009.

[10] H. Shaban, H. Elmikaty, and A. A. Shaalan, "Study the effects of electromagnetic band-gap (EBG) substrate on two patch microstrip antenna," Progress in Electromagnetics Research B, vol. 10, pp. 55-74, 2008.

[11] F. Yang and Y. Rahmat-Samii, "Reflection phase characterizations of the EBG ground plane for low profile wire antenna," IEEE Transactions on Antennas and Propagation, vol. 51, no. 10, pp. 2691-2703, 2003.

[12] J. R. Sohn, K. Y. Kim, H. S. Tae, and J. H. Lee, "Comparative study on various artificial magnetic conductors for low-profile antenna," Progress in Electromagnetics Research, vol. 61, pp. 2737, 2006.

[13] S. Chaimool, K. L. Chung, and P. Akkaraekthalin, "Bandwidth and gain enhancement of microstrip patch antennas using reflective metasurface," IEICE Transactions on Communications, vol. E93-B, no. 10, pp. 2496-2503, 2010.

[14] J. Liang and H. Y. D. Yang, "Radiation characteristics of a microstrip patch over an electromagnetic bandgap surface,"
IEEE Transactions on Antennas and Propagation, vol. 55, no. 6, pp. 1691-1697, 2007.

[15] G. Poilasne, "Antennas on High impedance ground planes: on the importance of the antenna isolation," Progress in Electromagnetics Research, vol. 41, pp. 237-255, 2003.

[16] S. Zhu and R. Langley, "Dual-band wearable textile antenna on an EBG substrate," IEEE Transactions on Antennas and Propagation, vol. 57, no. 4, pp. 926-935, 2009.

[17] M. Mantash, A. C. Tarot, S. Collardey, and K. Mahdjoubi, "Dual-band antenna for WLAN application with EBG," in the 4th International Congress on Advanced Electromagnetic Materials in Microwaves and Optics, pp. 794-796, Karlsruhe, Germany, September 2010.

[18] M. Mantash, A. C. Tarot, S. Collardey, and K. Mahdjoubi, "Dual-band CPW-fed G-antenna using an EBG structure," in Antennas and Propagation Conference (LAPC), pp. 453-456, Loughborough, UK, 2010.

[19] “ADS Momentum EM simulator tool," http://www.home .agilent.com/agilent/product.jspx? cc=EG\&lc=eng\&ckey= $1475688 \&$ nid $=-34360.0 .00 \& i d=1475688$.

[20] M. E. De Cos, Y. Álvarez, R. C. Hadarig, and F. Las-Heras, "Novel SHF-band uniplanar artificial magnetic conductor," IEEE Antennas and Wireless Propagation Letters, vol. 9, pp. 4447, 2010 .

[21] L. Yang, M. Fan, F. Chen, J. She, and Z. Feng, "A novel compact electromagnetic-bandgap (EBG) structure and its applications for microwave circuits," IEEE Transactions on Microwave Theory and Techniques, vol. 53, no. 1, pp. 183-189, 2005.

[22] A. Aminian, F. Yang, and Y. Rahmat-Samii, "In-phase reflection and EM wave suppression characteristics of electromagnetic band gap ground planes," in IEEE International Antennas and Propagation Symposium and USNC/CNC/URSI North American Radio Science Meeting, pp. 430-433, June 2003.

[23] R. C. Hadarig, M. E. De Cos, Y. ÁLvarez, and F. Las-Heras, "Novel bow-tie antenna on artificial magnetic conductor for $5.8 \mathrm{GHz}$ radio frequency identification tags usable with metallic objects," IET Microwaves, Antennas and Propagation, vol. 5, no. 9, pp. 1097-1102, 2011.

[24] M. E. De Cos, Y. Álvarez, and F. Las-Heras, "Enhancing patch antenna bandwidth by means of uniplanar EBG-AMC," Microwave and Optical Technology Letters, vol. 53, no. 6, pp. 1372-1377, 2011. 

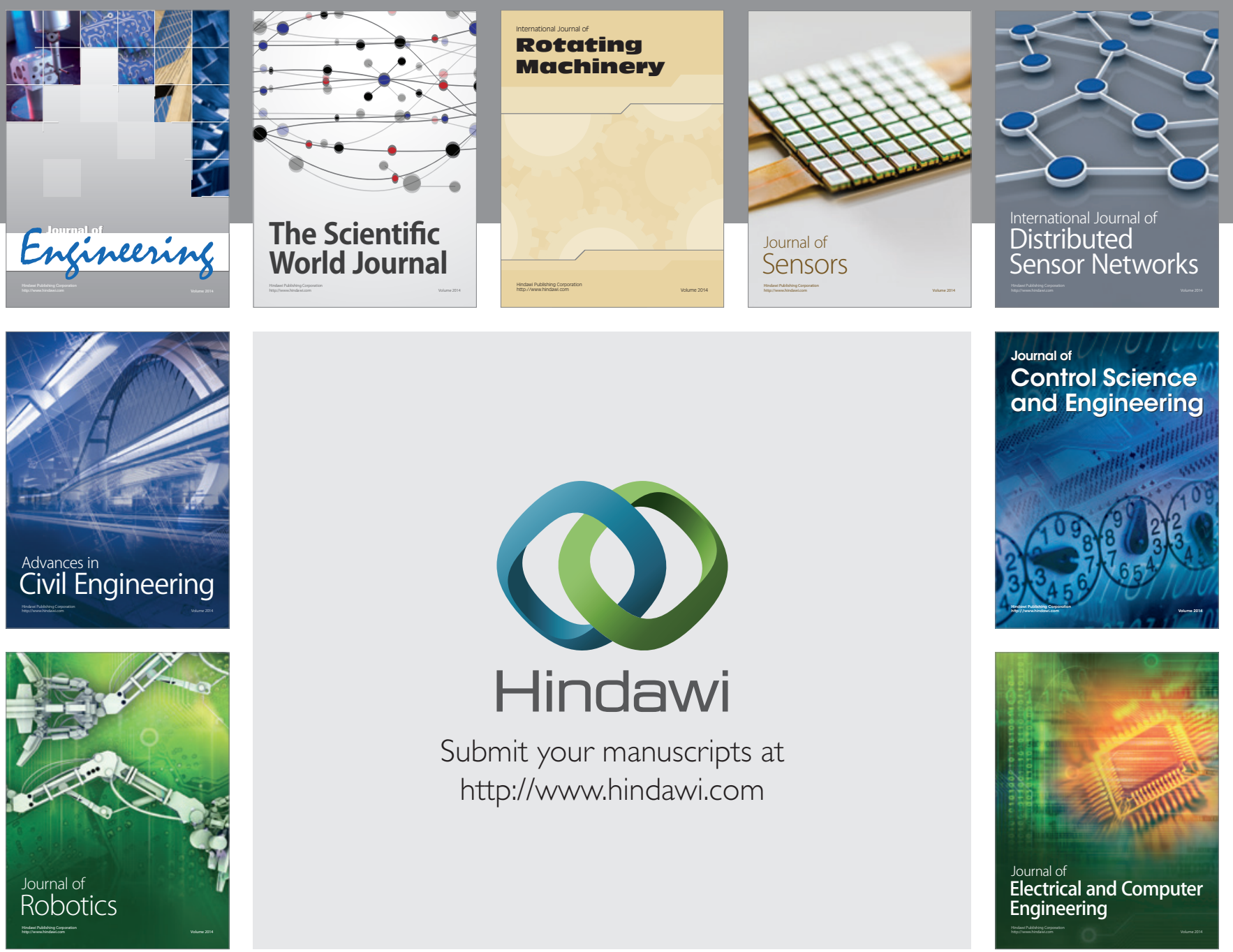

Submit your manuscripts at

http://www.hindawi.com
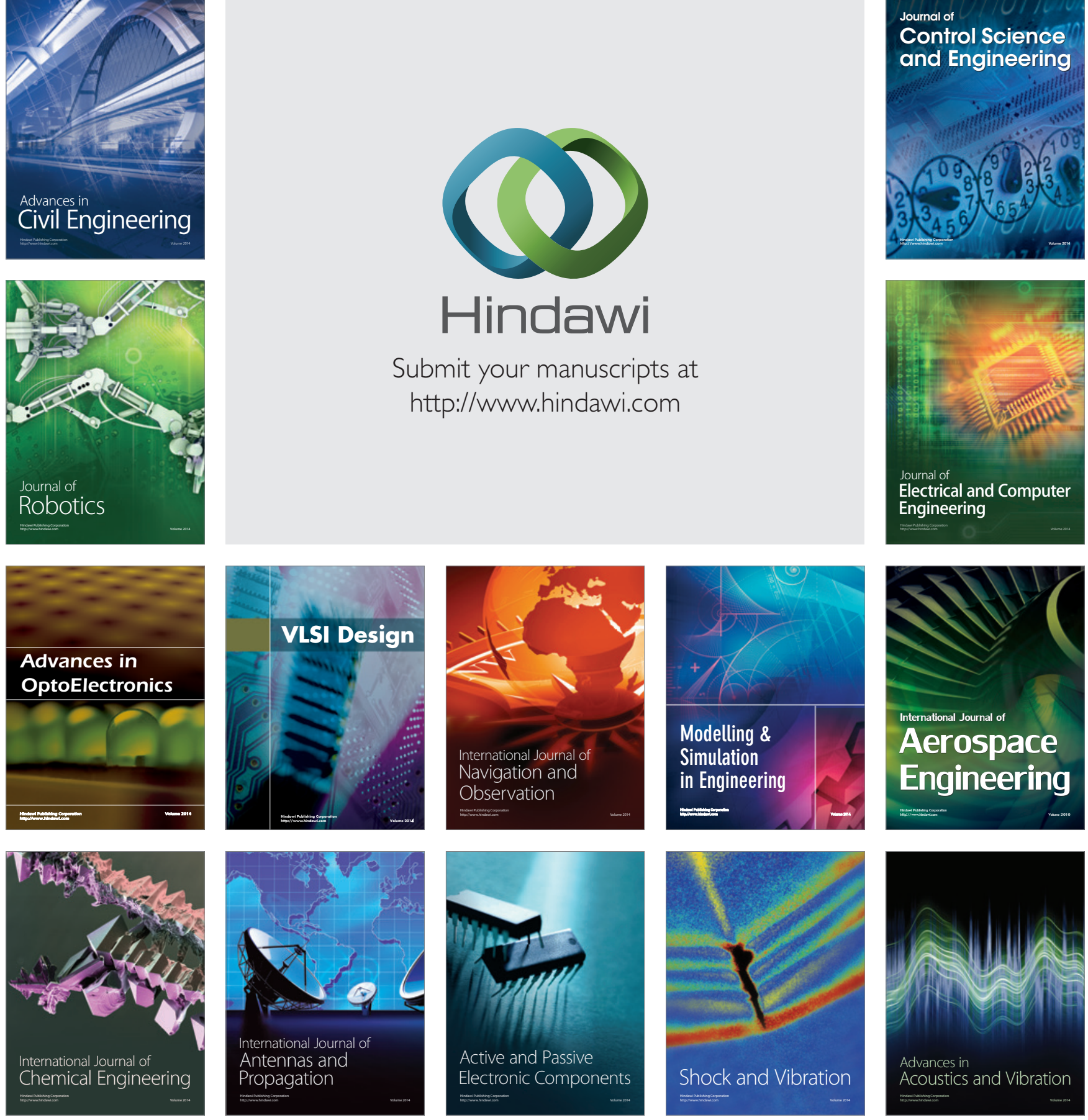